\title{
Toque: ferramenta terapêutica no tratamento geriátrico e gerontológico
}

\author{
Renata Francioni Lopes", Mirian Sales Soares", Lincon Féres de Sá***, Vilma Duarte Câmara****
}

\section{Resumo}

Trata-se de um estudo bibliográfico de caráter descritivo, realizado por meio de revisão de literatura. Foram utilizados como fontes artigos e resumos das bases de dados de bibliotecas virtuais, com os objetivos de apresentar os benefícios do toque como ferramenta eficaz para promoção do bem-estar em idosos e valorizar o toque no tratamento geriátrico e gerontológico. Os estudos analisados revelaram que o toque tem se mostrado uma ferramenta eficaz no tratamento de idosos, principalmente, naqueles que apresentam síndromes demenciais como alzheimer, e ainda, nas patologias como o câncer e outras. Segundo as pesquisas, alguns idosos relataram sensação de relaxamento e redução da ansiedade, produzindo bem-estar e conforto. No entanto, é necessário que os profissionais de saúde tenham interesse em conhecer os aspectos multifatoriais que envolvem os idosos, para que o toque seja uma "atitude solidária", realizado com afetividade, comprometimento, ética e profissionalis- mo, que garanta a promoção do bem-estar e um atendimento de qualidade aos idosos. Sugere-se, assim, a inserção do toque de forma mais ampla no contexto de pesquisa e na prática baseada em evidências, criando a possibilidade de desenvolver uma metodologia à sua aplicação na promoção da saúde e melhora da qualidade de vida dos idosos.

Palavras-chave: Toque terapêutico. Idosos. Programas de bem-estar. Profissional de saúde.

* Doutora em Endocrinologia pela Universidade Federal do Rio de Janeiro. Geriatra do Hospital Universitário Antônio Pedro, Campus Mequinho, da Universidade Federal Fluminense. Endereço para correspondência: Renata Francioni Lopes, Rua Passos da Pátria 105/101, bairro São Domingos, Niterói - RJ, CEP 24210-240. E-mail: renfrancioni@gmail.com

** Enfermeira. Especialista em Gerontologia e Geriatria Interdisciplinar pela Universidade Federal Fluminense.

**** Enfermeiro do Hospital Universitário Pedro Ernesto da Universidade Federal do Rio de Janeiro.

**** Doutora em Neurologia pela Universidade Federal do Rio de Janeiro. Professora Associada II de Neurologia e coordenadora do Programa de Geriatria e Gerontologia Interdisciplinar do Hospital Universitário Antônio Pedro, Campus Mequinho, da Universidade Federal Fluminense.

$\longrightarrow$ Recebido em março de 2009 - Avaliado em março de 2010.

$\hookrightarrow$ doi:10.5335/rbceh.2009.039 


\section{Introdução}

O envelhecimento populacional é um fenômeno crescente nos países desenvolvidos e em desenvolvimento, como o Brasil. A longevidade deve-se à redução da natalidade e ao aumento da expectativa de vida. Os problemas gerados pelo crescimento da população idosa provocam impactos sobre os setores econômico, social e da saúde, por repercutirem na aplicação dos recursos disponíveis para atender à demanda desses setores.

Com o fenômeno da globalização surgiu um extraordinário aparato tecnológico, facilitando os meios de comunicação a distância. Contudo, o quadro social que se percebe é de distanciamento, isolamento, segregação e consequente solidão, visto que a valorização da individualidade e competitividade são fatores ainda predominantes na sociedade. Ainda assim, observa-se que as pessoas estão propensas a buscar a melhora da qualidade para suas vidas, e é na população de idosos e dos que estão próximos a entrar na terceira idade que há um maior engajamento nesse sentido.

Portanto, há idosos que, mediante a realidade social ainda pouco estimulante, vêm buscando alternativas, como as terapias que utilizam o toque, incluindo massagens, técnicas de relaxamento, entre outras. Os idosos podem desenvolver processos patológicos e muitos deles, doenças crônico-degenerativas. Assim, observa-se que alguns idosos têm maior ou menor dificuldade diante das transformações biológicas de um processo que, embora progressivo, é perfeitamente natural. $\mathrm{O}$ toque alivia o desconforto e promove um estado de bem-estar, que contribui positivamente com o tratamento clínico.

O toque é amplamente utilizado por profissionais das diversas áreas de saúde e pode ter diferentes conotações para cada indivíduo, por se relacionar com os aspectos culturais, de conceitos e de impressões pessoais; é um importante meio de comunicação não verbal. Para quem o recebe, pode transmitir sensações de alívio, acolhimento, segurança e bem-estar. Entretanto, há pessoas que podem percebê-lo como uma "invasão da intimidade". Na verdade, quando bem direcionado, pode ser uma ferramenta terapêutica efetiva, por lidar com aspectos como as sensações no âmbito das emoções e do afeto. Pode-se, assim, aumentar o bem-estar.

É de suma importância ampliar conhecimentos e aprofundar reflexões sobre o toque e suas propriedades terapêuticas no cuidado com os idosos, uma vez que todos os profissionais de saúde devem buscar compreender as dimensões que abrangem o ato de tocar, direcionando esforços para a criação de uma metodologia a ser incorporada ao modelo biológico existente. $\mathrm{O}$ cuidado técnico se associa ao cuidado subjetivo, e este é aspecto relevante no processo saúde-doença. Assim, este estudo pretende apresentar os aspectos que envolvem o toque como ferramenta terapêutica eficaz para a promoção do bem-estar em idosos, valorizando-o no tratamento geriátrico e gerontológico. 


\section{Metodologia}

Trata-se de um estudo bibliográfico de caráter descritivo, realizado por meio da revisão da literatura. Foram realizados a leitura, a seleção, o fichamento e o arquivo dos tópicos de interesse para a pesquisa em pauta, com a finalidade de conhecer as contribuições científicas existentes sobre o toque terapêutico. Foram utilizados como fonte de dados os artigos e resumos disponíveis nas bases das bibliotecas virtuais, Lilacs, Medline e Pubmed, incluindo ainda, revistas indexadas e livros sobre a temática do estudo.

\section{Referencial teórico}

\section{0 toque}

$\mathrm{O}$ ato de tocar é uma das formas mais significativas de estabelecer contato e, ainda, de expressar sentimentos quando a palavra verbalizada não é eficaz o suficiente, ou não está presente para traduzir o que se intenciona ao outro, como nos casos de afasia em idosos demenciados, ou mesmo na presença de mecanismos de adaptação em face da doença, como negação, raiva, rebeldia, isolamento, etc.

Quando o silêncio se impõe, o toque é fundamental para nos comunicarmos com os que cuidamos, demonstrando-lhe que essa nos importa, que nos preocupamos com ela, não apenas com a sua doença. $\mathrm{O}$ toque tem múltiplas funções: pode servir para fazer notar a presença de alguém, para cumprimentar, chamar a atenção e confortar, enfim, toda vivência humana está intimamente associada ao toque como forma de comunicação. (PACHECO; VIEGAS; ROSA, 2007).
O toque pode ter inúmeras representações para cada indivíduo, dependendo do contexto sociocultural em que esteja inserido. Assim, fatores como costumes, tabus, e componentes psicossociais têm influência preponderante na maior ou menor aceitação do toque. Entretanto, segundo Pacheco, Viegas e Rosa (2007), todos precisamos do toque, desde as crianças, para estabelecer relações e sobreviver, idosos, que pelo toque podem minimizar a sensação de solidão ou de que não são queridos.

\section{Características}

Identificado como maior órgão que compõe o ser humano, a pele pode ser objeto de relevância quanto à sua utilização para a promoção do bem-estar e melhora da qualidade de vida das pessoas, principalmente, os que são referência deste estudo - os idosos.

Falar de tato é falar de pele. A pele que nos cobre é como um envelope gigante, um órgão que recebe impressões táteis ou sensoriais e que reage a qualquer contato com sensações específicas; seus receptores reagem a calor, frio, toque, coceira, cócegas, tipos de dor e vibrações. Esse órgão compreende aproximadamente $20 \%$ de nosso peso corporal, com milhões de minúsculos terminais nervosos que atuam como transmissores de sensações, enviando mensagens sob diversas formas, cujo sentido se busca compreender. O poder do toque está relacionado à importância da pele, que constitui uma verdadeira fronteira pela qual passa uma grande parte das relações entre cada ser e o seu ambiente físico, biológico e afetivo. (DAVIS, 1991). 
Destaca-se, assim, a pele como um instrumento essencial no enfoque das terapias alternativas que envolvem $o$ toque, bem como a necessidade de ampliar os estudos e pesquisas científicas relacionadas ao tema.

Segundo Pereira e Pereira (2002), a estimulação tátil tem efeitos profundos sobre o organismo do indivíduo, sendo a pele fonte imunológica de hormônios para a diferenciação de células protetoras, desempenhando um papel de destaque na regulação da pressão e fluxo sanguíneo. Citam ainda, dentre as funções relacionadas ao toque, as de sediar receptores sensoriais e a de mediar sensações. Todas essas funções podem favorecer o estado de saúde do indivíduo, já que, ao acionar os receptores sensoriais, o toque pode produzir emoções mais reconhecidamente benéficas no enfrentamento e recuperação de doenças.

\section{Tipologias}

A literatura descreve alguns tipos de toque realizados em pacientes no atendimento hospitalar, ambulatorial, entre outros, com ênfase em três tipos: o toque instrumental, o afetivo e o instrumentalafetivo. Assim, o toque instrumental é entendido como aquele realizado numa tarefa ou procedimento específico (ex: aferir sinais vitais, exame físico); o toque afetivo, como contato espontâneo, visa demonstrar apoio, carinho e segurança (ex: nas situações de dor, medo, ansiedade, depressão, solidão); e toque instrumental-afetivo, como uma combinação entre os dois anteriores (ex: utiliza-se o componente afetivo de forma consciente na realização de qualquer procedimento). (GALA; TELLES; SILVA, 2003; PINHEIRO; ROCHA; SILVA 1998).

\section{0 idoso e 0 toque}

Os idosos são adultos que, entrando em nova fase, precisam se adaptar às transformações impostas por mais uma etapa da vida. As características pessoais de cada indivíduo no processo de envelhecer o influenciarão de forma positiva, ou negativa, dependendo do seu estado de espírito, da crenças, disposição para o enfrentamento nas situações adversas, condições socioeconômicas e culturais, etc.

A longevidade é característica do mundo atual e apresenta-se demograficamente crescente num nível sem precedentes. Segundo a Organização Mundial da Saúde, as projeções para o ano de 2025 apontam o Brasil como o sexto país com o maior número de pessoas com sessenta anos ou mais, ou seja, cerca de 32 milhões de pessoas idosas. (KALACHE; VERAS; RAMOS, 1987).

É relevante considerar as questões que abrangem o processo de envelhecimento; desconsiderá-las seria o mesmo que ignorar toda a essência que envolve o indivíduo na etapa da senectude. Portanto, no tocante ao envelhecer, é imperioso que se dê atenção ao aspecto psíquico. Maffioletti (2005) comenta que a psicologia vem sustentando significações que ao longo dos anos vêm contribuindo com material para a construção e manutenção dos esteriótipos e da crença na existência de um "envelhecimento psíquico".

Em sua pesquisa sobre a velhice e família, Maffioletti (2005) refere-se ao 
envelhecimento orgânico como estimulador de investigações sobre as alterações cognitivas e comportamentais. Trazem, assim, a contribuição para se caracterizarem os estágios da vida, gerando uma espécie de compreensão ordinária dos processos psíquicos que decorreriam da utilização de uma causalidade mecânica, supondo que exista, decorrente do envelhecimento, uma forma de sentir e entender o mundo que é universal.

$\mathrm{O}$ toque pode aliviar as tensões advindas das frustrações, de desapontamentos, que causam dor e desalento a muitos dos que envelhecem sem a perspectiva de um lar, ou, ainda quando nele, vivenciam o distanciamento daqueles que o amparam, cuidam e afagam. Existem ainda inúmeras situações que comprometem a autonomia do idoso, conferindo a estes certas limitações, com prejuízo para a realização de suas atividades de vida diária, como, por exemplo, deambular, vestir-se e alimentar-se. Os idosos estão mais expostos aos riscos de quedas, com consequentes danos físicos e psicológicos, que vão desde as fraturas, imobilidade e aumento do risco de institucionalização, ao medo de sofrer novas quedas e, também, o risco de morte. $\mathrm{O}$ toque em idosos hospitalizados pode minimizar algum sofrimento. (SILVA; DOMINGUEZ, 1996, 1997).

\section{0 toque na promoção do bem- estar}

\section{Bem estar}

Observa-se na população idosa que os efeitos do toque integram os aspectos mais positivos e amplos relacionados ao bem-estar. Portanto, bem-estar significa conforto; tranquilidade. Ainda, de forma mais ampla, é definido como um estado dinâmico de saúde no qual o indivíduo progride em direção a um nível elevado de funcionalidade, atingindo um equilíbrio ótimo entre o meio interno e o externo. (PEIXOTO, 2007).

Assim, diante das situações críticas e das adversidades que são fatores geradores do estresse para as pessoas, caracteriza-se como bem-estar o equilíbrio entre os sentimentos positivos e negativos, visto que se manter calmo e confiante o bastante para enfrentar as "batalhas diárias" travadas no cotidiano, parece ser o caminho para aumentar a qualidade de saúde e de vida. Assim, o toque é um instrumento e uma atitude solidária com relação àqueles que já travaram muitas dessas "batalhas" em sua jornada de vida, necessitando, por isso, de atenção, proteção, carinho e segurança para uma vida mais plena e feliz.

\section{Bem-estar subjetivo}

O bem-estar subjetivo (BES) é uma área da psicologia que tem crescido reconhecidamente nos últimos tempos e cobre estudos que têm sido utilizados com diversas nomenclaturas, tais como satisfação com a vida, felicidade, estado de espírito e afeto positivo. Além disso, o BES é considerada uma avaliação subjetiva da qualidade de vida, referindo-se ao que as pessoas pensam e como elas se sentem sobre as suas vidas. Atualmente, há perspectivas que o definem como uma ampla categoria de fenômenos, que incluam as respostas emocionais das 
pessoas, domínios de satisfação e os julgamentos globais de satisfação de vida. (ALBUQUERQUE; TRÓCCOLI, 2004)

Estudos com relação ao envelhecimento mostram que o bem-estar subjetivo está associado a um envelhecimento mais saudável, quando envolve aspectos emocionais tanto positivos como negativos, em razão dos estados depressivos e de doenças físicas. Destacam ainda que o bem-estar subjetivo é um importante indicador do nível de adaptação de idosos, estágio do desenvolvimento que já corresponde, na estrutura demográfica atual, a aproximadamente $25 \%$ da duração de vida das pessoas e que se constitui de componentes afetivos e cognitivos. (GUEDEA et al., 2006).

Assim, o bem-estar subjetivo pode ser considerado um dos indicadores de saúde mental. Entretanto, distinguese de saúde psicológica propriamente dita por se caracterizar apenas com um aspecto do bem-estar psicológico, que, embora necessário, é insuficiente para indicar que a pessoa esteja bem com sua vida. As pessoas podem estar bem em vários aspectos de suas vidas, mas não se sentirem felizes, ao passo que outras podem ser ou estar em condição de insanidade mental, mas se sentirem felizes. (ALBUQUERQUE; TRÓCCOLI, 2004).

Há poucas medidas validadas de bem-estar subjetivo adaptadas à população brasileira e segundo alguns autores, existem pontos de discordância teórica relativos à conceituação do BES. Contudo, há um consenso entre algumas dimensões, como satisfação com a vida, afetos positivos e negativos. O elemento subjetivo é essencial na avaliação da qualidade de vida de um indivíduo ou de um grupo, pois os indicadores sociais, por si só, não seriam capazes de defini-la, já que as pessoas reagem diferentemente a circunstâncias semelhantes.

\section{Relação idoso-profissional de saúde}

Um aspecto fundamental para o estabelecimento de uma boa relação profissional-idoso é a troca de informações. Cada vez mais as pessoas querem se encarregar de decisões sobre seu tratamento, buscando nos profissionais responsáveis seus conselheiros. Assim, observa-se que a relação idoso-profissional de saúde deve ser construída e consolidada com base em confiança e empatia.

A relação idoso-profissional de saúde pode se apresentar de maneiras variadas, com características como rápida, insensível, grave, solene, ou ainda, compreensiva, genuína, afetiva e equilibrada. Sem dúvida, a relação que abrange os últimos aspectos citados se faz mais adequada, principalmente nas situações em que o paciente terá o impacto de uma notícia que para ele pode ser desagradável e triste. (PIRES, 2008).

O profissional de saúde tem a tarefa de prestar uma assistência de qualidade, que consiste no cumprimento do dever ético-moral que jurou cumprir ao abraçar a profissão na área de saúde. A fidelidade é o dever de lealdade e compromisso do profissional para com o paciente, que serve de base entre ambos. A utilização verdadeira e honesta das informações é 
um dever primário do profissional e alicerce dessa fidelidade. (GOLDIN, 1998).

O idoso necessita de maior atenção e, geralmente, de cuidados mais específicos em razão das transformações características do processo de envelhecimento. Essas mudanças envolvem aspectos multifatoriais, pois, diante das doenças que preexistem e se exacerbam, ou das que surgem no decorrer desse processo, criam circunstâncias de dependência, com necessidades de adaptação tanto para idoso quanto para seus familiares. No Brasil, a prestação de serviços na área de saúde é oferecida em meio a limitações e dificuldades que se refletem no atendimento, comprometendo a qualidade da assistência prestada. Portanto, na prática, a terapêutica com os idosos requer um conhecimento amplo, que englobe todos os aspectos a eles relacionados no contexto biopsicossocial.

O toque é um meio de comunicação que pode ser utilizado como uma excelente ferramenta na terapêutica com idosos, principalmente aqueles em situação de abandono, acometidos por processos patológicos físicos e mentais, internados em unidades hospitalares, ou mesmo em suas residências, por ser capaz de lhes transmitir sentimentos de apoio, carinho, segurança, proteção, promovendo um estado de bem-estar.

\section{Análise e discussão}

Estudos clínicos que confirmam o toque como ferramenta terapêutica eficaz para a promoção do bem-estar em idosos apontam a importância do toque no tratamento geriátrico e gerontológico.
Holland e Pokorny (2001) realizaram um estudo experimental com aplicação de massagens durante três dias consecutivos em 24 pacientes que haviam sofrido acidente vascular cerebral (AVC) leve, com faixa etária entre 52 e 88 anos, e média de 71,8 anos, em Unidade Hospitalar da Carolina do Norte - EUA. Utilizaram um instrumento para avaliar a resposta fisiológica e psicológica, denominado Huckstadt touch. Os resultados apontaram uma redução significativa na média de frequência cardíaca e respiratória no primeiro e terceiro dia, e os pacientes relataram sentimentos de bem-estar, como relaxamento, calma, felicidade e um sentimento de proximidade com as enfermeiras, que os faziam se sentir cuidados e acolhidos, diminuindo o grau de ansiedade.

Davis, em seu livro $O$ poder do toque, relata que inúmeros profissionais médicos e enfermeiros afirmam que $o$ contato físico é um dos aspectos mais importantes da reabilitação, seja nos ferimentos traumáticos, seja nas enfermidades agudas, e "que de nada vale todos os remédios e recursos materiais, se o paciente não desejar viver". Destaca ainda que os sentimentos de amor têm consequências físicas; que o contato físico que transmite amor pode desencadear alterações metabólicas e químicas no corpo, as quais auxiliam na cura, e que a estimulação tátil e as emoções positivas podem controlar a endorfina, que alivia a dor, proporcionando uma sensação de bem-estar. (DAVIS, 1991).

O profissional de saúde deve se interessar em conhecer e compreender os estágios que envolvem o idoso diante 
das situações ameaçadoras à sua saúde física e/ou mental. Essa compreensão ajudará o profissional a entender melhor os sentimentos vivenciados pelos idosos, o que contribuirá para que estes sejam tratados de maneira mais adequada, especialmente nos "momentos críticos".

Ao tocar ou segurar a mão dos idosos com respeito e carinho, nós, profissionais de saúde, contribuímos para tranquilizálos e encorajá-los, traduzindo por meio dos gestos que nos importamos com eles e que são bem-vindos. Essa "atitude solidária”, certamente, faz muita diferença, o que se confirma por alguns estudos relacionados a seguir.

Weze et al. (2004) realizaram um estudo clínico, randomizado, prospectivo e controlado no Centro de Atendimento Complementar em Eskdale, Cumbria Escócia, no período entre 1995 e 2001, do qual participaram 35 pacientes com câncer, com idade média de oitenta anos, de ambos os sexos, para avaliar os efeitos do toque suave do pré para $o$ pós-tratamento e mudanças no funcionamento físico e psicológico. As avaliações foram feitas por meio de um questionário com escala visual analógica para a classificação subjetiva dos sintomas e com base no EuroQol (EQ-5D), uma medida genérica do estado de saúde validada pelo Wilcoxon Ranks Testes. Os resultados revelaram que, estatisticamente, houve significativas melhorias no funcionamento físico e psicológico, com efeitos positivos na qualidade de vida; melhorias acentuadas foram observadas em avaliações de estresse e relaxamento, da dor, desconforto e depressão. $\mathrm{O}$ estudo não encontrou qualquer efeito negativo resultante do toque suave como tratamento, o qual foi seguro e eficaz para auxiliar no tratamento médico convencional, com potencial para melhorar alguns aspectos mais estressantes do câncer e de seu tratamento.

Malaquin-Pavan (1997) realizou um ensaio clínico no Hospital Corentin Celton, França, no qual, quatro idosos que apresentavam défices cognitivos e alterações características de demência de Alzheimer, com comportamento anormal, como agitação, gritos, agnosia, ataxia e apatia, foram submetidos a duas sessões semanais de toque-massagem, ou massagem terapêutica durante $30 \mathrm{~min}$ cada um, por seis meses, significando 51 sessões para cada um, num total de 204 sessões.

O estudo aborda os efeitos do toquemassagem no comportamento dos idosos a fim de verificar as reações intrapessoais, verbalizadas ou não pelos idosos durante as sessões. Os resultados são apresentados segundo os aspectos coletivos, porém o estudo avaliou as reações individuais e as comparou, estabelecendo, assim, resultados expressos pelo grupo de idosos. A atividade foi aceita em $95 \%$. Durante os 30min de sessão foram observados sinais físicos diferentes, como relaxamento (59\%), sonolência (34\%), diferentes maneiras de estabelecer comunicação com os enfermeiros (59\%), fim ou redução do comportamento anormal (79\%). Esses resultados provam o real benefício do toque-massagem, o que traz possibilidades para o tratamento das aflições vivenciadas pelo idoso.

Silva e Domingues (1997) realizaram um estudo com o objetivo de averiguar 
a percepção dos idosos a respeito do toque, por meio de um questionário com perguntas sobre como e quando ocorre o toque, sentimentos que desperta, entre outros. Participaram da pesquisa cerca de vinte idosos hospitalizados. Os principais resultados revelaram que a maioria dos idosos sente bem-estar quando são tocados; concordam que há predominância do toque instrumental e consideram importante a maneira como se toca.

Durante o processo de leitura e seleção dos artigos e revistas para essa pesquisa, foi encontrado um número considerável de estudos realizados que utilizaram o toque terapêutico "sem o toque", com técnica não invasiva, baseado no conceito de campo energético, utilizado por muitos como uma ferramenta terapêutica. (WOODS; DIMOND, 2002; SMITH et al., 2003; VENTEGODT; MORAD; MERRICK, 2004; WOODS; CRAVEN; WHITNEY, 2005).

Essa técnica, dita contemporânea, por se basear na imposição das mãos, uma antiga arte, com o registro de sua prática desde 1552 A.C., foi difundida pela professora Krieger, da Escola de Enfermagem de Nova York - EUA e pela terapeuta Kunz. Ambas desenvolveram a disciplina que chamaram "O toque Terapêutico", para ajudar os pacientes a melhorar a saúde física e emocional, ficando conhecido mais tarde como método Krieger-Kunz de toque terapêutico. (PACHECO; VIEGAS; ROSA, 2007). No entanto, a abordagem do presente estudo refere-se ao "toque-tato", com enfoque no contato físico, apontado na presente pesquisa como um elemento essencial na relação entre as pessoas; portanto, indispensável no tratamento geriátrico e gerontológico.

$\mathrm{O}$ toque pode ser uma ferramenta poderosa e eficaz ao alcance de todos os profissionais de saúde. (GALA; TELLES; SILVA, 2003). Entretanto, deve ter sua utilização direcionada e baseada em parâmetros como compromisso, ética profissional e metodologia, somados a sentimentos humanitários de afetividade e solidariedade, que podem e devem ser estimulados em todos os que atuam com os idosos na área de saúde. Assim, a criação de espaços intra e extra-hospitalares para realização de dinâmicas de grupo, terapias alternativas que utilizem o toque e grupos de pesquisa sobre o toque e sua relação com o processo de envelhecimento são recursos que podem auxiliar os profissionais menos familiarizados com o contexto humanístico a desenvolver as habilidades afetivas, sendo muito útil à prática médica global e aos multiprofissionais que atuam no campo da geriatria e gerontologia.

\section{Considerações finais}

$\mathrm{O}$ toque no tratamento geriátrico e gerontológico é uma atitude solidária que envolve comprometimento e afetividade, não sendo apenas um ato mecânico. O toque tem se mostrado uma eficaz e valiosa ferramenta na terapêutica com idosos.

Mediante a manutenção de aspectos psicoafetivos e sociais, já muitas vezes comprometidos por circunstâncias da vida, os que alcançam a velhice necessitam ainda de maior respeito, de carinho, e de segurança que lhes garantam um viver com qualidade.

RBCEH, Passo Fundo, v. 6, n. 3, p. 402-412, set./dez. 2009 
Touch: therapeutic tool in geriatry and gerontology treatment

\section{Abstract}

This is a bibliographic study of descriptive character realized by literature's revision. It was used articles and summaries from the data basis of the virtual libraries as sources. Their objectives are to present the benefits of the Touch as effective tool to promote well-being of the elderly and to value the touch in geriatrics and gerontology treatment. The analyzed studies revealed that the touch has seemed an effective tool in treatment of the old persons, mainly in those who present dementia symptoms as Alzheimer's disease and pathologies, as cancer etc. According to researches, some elderly related feeling of relaxation and a reduction in anxiety, which promoted feeling of well-being and comfort. However it's necessary that health professionals have interest in knowing the many factors and aspects that involve the elderly in order to become the touch a "solidarity attitude", done with emotionally, commitment, ethics and directive to guarantee the promotion of well-being and a quality attendance to the elderly. It's also suggested the touch is widespread in the research context and in the practice, based on evidences, therefore creating possibilities of developing a methodology for its application in the promotion of health and improvement in the life quality of the elderly.

Key words: Therapeutic touch. Aged. Health promotion. Health professional.

\section{Referências}

ALBUQUERQUE, A. S.; TRÓCCOLI, B. T. Desenvolvimento de uma escala de bem-estar subjetivo. Psicologia - Teoria e Pesquisa, Brasília, v. 20, n. 2, p. 479-484, maio/ago. 2004. Disponível em: <www.scielo.br/scielo. php?script=sci $>$. Acesso em: 18 jul. 2008.
DAVIS, P. K. O poder do toque. São Paulo: Best Seller, 1991.

GALA, M. F.; TELLES, S. C. R.; SILVA, M. J. P. Ocorrência e significado do toque entre profissionais de enfermagem e pacientes de uma UTI e unidade semi-intensiva cirúrgica. Revista da Escola de Enfermagem da Universidade de São Paulo, São Paulo, v. 37, n. 1, p. 52-61, 2003.

GOLDIN, J. R. Bioética. 1998. Disponível em: <www.ufrgs.br/bioetica/relido.htm>. Acesso em: 18 jul. 2008.

GORDON, A. et al. The effects of therapeutic touch on patients with osteoarthritis of the knee. J. Fam. Pract., v. 47, n. 4, p. 271-277, Oct. 1998.

GUEDEA, M. T. D. et al. Relação do bemestar subjetivo, estratégias de enfrentamento e apoio social em idosos. Psicologia - Reflexão e Crítica, Porto Alegre, v. 19, n. 2, p. 301308, 2006.

HOLLAND, B.; POKORNY, M. E. Slow stroke back massage: its effect on patients in a rehabilitation setting. Rehabil Nurs, v. 26, n. 5, p. 182-186, Sep./Oct. 2001.

KALACHE, A.; VERAS, R. P.; RAMOS, L. R. O envelhecimento da população mundial: um desafio novo. Revista de Saúde Pública, São Paulo, v. 21, n. 3, p. 200-210, 1987.

MAFFIOLETTI, V. L. R. Velhice e família: reflexões clínicas. Psicologia - Ciência e Profissão, Brasília, v. 25, n. 3, p. 336-351, set. 2005.

MALAQUIN-PAVAN, E. Bénéfice thérapeutique du toucher-massage dans la prise en charge globale de la personne âgée démente. Recherche en Soins Infirmiers, s. v., n. 49, p. 11-66, jun. 1997.

PACHECO, S. C. C.; VIEGAS, S. M. F. S. M.; ROSA, Z. M. M. Toque terapêutico: fundamentação e aplicabilidade em enfermagem. Revista Nursing Portuguesa, Lisboa, s. v., n. 224, set. 2007. Disponível em: <www.forumenfermagem.org >. Acesso em: 26 jul. 2008. 
PEIXOTO, T. Saúde e bem-estar. 2007. Disponível em: <www.spas-portugal.com>. Acesso em: 6 ago. 2008.

PEREIRA, C. M.; PEREIRA, L. S. O toque em crianças com câncer durante sessões de quimioterapia. CienteFico, Salvador, v. 1, n. 2, p. 1-4, ago./dez. 2002. Disponível em: <www.frb.br>. Acesso em: 6 ago. 2008.

PINHEIRO, E. M.; ROCHA, I. F.; SILVA, M. C. M. Identificação dos tipos de toque ocorridos no atendimento de enfermagem de um serviço ambulatorial. Revista da Escola de Enfermagem da Universidade de São Paulo, São Paulo, v. 32, n. 3, p. 192-198, out. 1998.

PIRES, A. P. Comunicação de más notícias. Serviço de psicologia/HCPA e Núcleo interinstitucional de bioética. Disponível em: $<$ www.ufrgs.br/bioetica/masnot.htm $>$. Acesso em: 14 set. 2008

SILVA, M. J. P.; DOMINGUES, J. Idosos asilados: como percebem o toque? O Mundo da Saúde, São Paulo, v. 20, n. 9, p. 298-306, out. 1996.

. O toque: percepção de idosos hospitalizados. Texto \& Contexto em Enfermagem, Florianópolis, v. 6, n. 2, p. 291-299, maio/ ago. 1997.

SMITH, M. C. et al. Outcomes of touch therapies during bone marrow transplant. Altern. Ther. Health Med., n. 9, v. 1, p. 40-49, Jan./ Feb. 2003.

VENTEGODT, S.; MORAD, M.; MERRICK, J. Clinical holistic medicine: classic art of healing or the therapeutic touch. The Scientific Word Journal, v. 4, n. 3, p. 134-147, Mar. 2004.

WEZE, C. et al. Evaluation of healing by gentle touch in 35 clients with cancer. Eur. J. Oncol. Nurs., v. 8, n. 1, p. 40-49, Mar. 2004.

WOODS, D. L.; CRAVEN, R. F.; WHITNEY, J. The effect of Therapeutic touch on behavioral symptoms of persons with dementia. Altern. Ther. Health Med., v. 11, n. 1, p. 66-74, Jan/Feb. 2005.
WOODS, D. L.; DIMOND, M. The effect of therapeutic touch on agited behavior an cortisol in persons with Alzheimer's disease. Biol. Res. Nurs., v. 4, n. 2, p. 104-114, Oct. 2002. 